\title{
Integrated proteomics and biological assays for characterization of non-nutritional effects of butyric acid on intestinal cells*
}

\author{
S. Purup ${ }^{1,3}$ and L.B. Larsen ${ }^{2}$ \\ University of Aarhus, Faculty of Agricultural Sciences, \\ ${ }^{1}$ Department of Animal Health, Welfare and Nutrition, \\ ${ }^{2}$ Department of Food Science \\ P.O. Box 50, DK-8830 Tjele, Denmark
}

\begin{abstract}
The non-nutritional effects of butyric acid on human intestinal epithelial cells were characterized by integration of biological assays and 2-dimensional gel-based proteomics for characterization of cellular protein expression. Human colonic adenocarcinoma (Caco-2) cells were cultured with sodium butyrate $(\mathrm{NaB})$, foetal calf serum (fast-growing cells) or basal medium alone (control cells). Biological assays showed that butyric acid decreased cell proliferation and induced apoptosis. Principal component analysis of proteomic results showed that protein expression in NaB-treated cells was different from both control and fast-growing cells. Significantly different expressed proteins were characterized by mass spectrometry.
\end{abstract}

KEY WORDS: butyrate, milk, cell proliferation, apoptosis, proteomics

\section{INTRODUCTION}

A number of nutritionally non-essential components present in the diet have been discovered to have beneficial effects for general health and in prevention of a number of diseases, including cancer. One such nutrient, the short chain fatty acid, butyric acid, can be derived in large quantities from bacterial fermentation of dietary fibre in the bowel, and is also a component of bovine milk (Jensen, 2002). Several animal studies have demonstrated that fibres producing the highest levels of butyrate in the colon are correlated with a greater protection against colon carcinogenesis (Archer and Hodin,

\footnotetext{
${ }^{*}$ Supported by the Danish Agency for Science, Technology and Innovation

${ }^{3}$ Corresponding author: e-mail: Stig.Purup@agrsci.dk
} 
1999). However, the cellular effects of butyrate are complex, especially since those observed in normal colon epithelial cells may be the opposite of those in transformed colon epithelial cells. In colon epithelial cells, proliferation may be stimulated by butyrate, while in transformed colon epithelial cells proliferation may be inhibited and differentiation promoted (Dzierzewicz et al., 2002).

The Caco-2 cell line is a well-established model of human colorectal tumour. It represents a selected population of colon cancer cells that have adapted to conditions in vitro, and have been widely used in studies of differentiation and regulation of intestinal functions.

Proteomics is a tool for the simultaneous determination of the protein composition of complex mixtures, like cell lysates. One of the tools in proteomics is to combine the separation power of two-dimensional gel electrophoresis (2DE) with multivariate statistics and mass spectrometry (MS) for identification of differentially expressed proteins in response to different treatments. The objective of this study was to investigate the non-nutritional effects of $\mathrm{NaB}$ on intestinal cells by integrated biological and proteomic methods.

\section{MATERIAL AND METHODS}

\section{Cell proliferation}

Human colonic adenocarcinoma (Caco-2) cells were cultured in Dulbecco's Minimal Essential Medium (DMEM with $25 \mathrm{mM}$ Hepes) supplemented with foetal calf serum (FCS, 10\%), L-glutamine ( $2 \mathrm{mM}$ ), and antibiotic solution for $24 \mathrm{~h}$ (pretreatment period). The treatments included medium containing FCS $(0.625 \%$; control cells), $\mathrm{NaB}$ in different concentrations $(0.1,1,10$ and $50 \mathrm{mM})$, or FCS (10\%; fast-growing cells) added for incubation periods of $48 \mathrm{~h}$. Cell proliferation was measured by alamarBlue reduction (BioSource International Inc.).

\section{Preparation of cell lysates for apoptosis and proteomics}

For measurement of cell apoptosis and for proteomic analysis of protein expression, lysates of Caco-2 cells were produced by addition of RIPA lysis buffer. The treatments included: medium containing FCS $(0.625 \%$; control cells), $10 \mathrm{mM} \mathrm{NaB}$ (apoptotic cells), or FCS (10\%; fast-growing cells) added for incubation periods of $48 \mathrm{~h}$. Cell lysates were frozen at $-80^{\circ} \mathrm{C}$ until proteomic analysis or measurement of apoptosis as caspase 3/7 activity (Promega Corporation). The cell lysates for proteomic studies were prepare from two different experiments: at day one two replicate gels of each of the three different treatments were analysed, while at day 2 four replicate gels of each treatment were used, giving a total of 18 gels in the experiment. 


\section{Two-dimensional gel electrophoresis}

The 2DE was carried out essentially as described by Lametsch and Bendixen (2001) with the following modifications. A Dodeca cell (BioRad) allowing the simultaneous analysis of 12 gels was used. The first dimension of protein separation was carried out in immobilized $11 \mathrm{~cm}$ IPG strips ( $\mathrm{pH}$ 5-8; BioRad), with $12.5 \%$ gradient Criterion gels (BioRad) in the second dimension. For analytical gels subjected to image analysis a volume of cell lysates corresponding to $50 \mu \mathrm{g}$ as determined by BCA assay (BioRad) was applied, whereas for preparative gels for MS analysis a volume of sample corresponding to $370 \mu \mathrm{g}$ protein was applied. Analytical gels were silver stained according to Lametsch and Bendixen (2001), while preparative gels for MS were stained according to Shevchenko et al. (1996).

\section{In gel digestion, desalting and concentration of protein spots}

Protein spots of significance were subjected to in-gel digestion by addition of trypsin essentially as described by Jensen et al. (1998), and the peptide mixture was desalted and concentrated as described by Lametsch et al. (2002). The peptides were eluted in $0.5 \mu 1$ matrix solution (15-20 g/L of $\alpha$-cyano-4-hydroxycinnamic acid in $70 \%$ acetonitrile) directly onto the MALDI target plate (Bruker Daltonics $\mathrm{GmBH})$.

\section{Identification of proteins by MALDI-TOF mass spectrometry}

Mass spectra were obtained using a Bruker Ultraflex MALDI-TOF mass spectrometer (Bruker Daltonik $\mathrm{GmBH}$ ) in reflection mode. The ion accelerating voltage was $25 \mathrm{kV}$ with a delay time of $40 \mathrm{~ns}$. The laser frequency was $50 \mathrm{~Hz}$ and 200 laser shots were accumulated for each spectrum. Mass searches were carried out in the data base Swiss Prot (Swiss Institute of Bioinformatics) using the MS ion search programme Mascot (Matrix Science).

\section{Image analysis and multivariate statistics}

The 2DE gels $(\mathrm{n}=18)$ were analysed using ImageMaster 2D analyser software (Amersham Pharmacia). The relative spot volumes were calculated for each separate gel to overcome gel-to-gel variations in spot intensities and sample loadings. Data was evaluated by principal component analysis (PCA) and discriminant PLS (D-PLS) using the multivariate statistical software package Unscrambler ver. 9.2 (Camo Process A/S). Standardized (centred: $\mu=0$, and normalized: $1 / \mathrm{SD}$ ) variables and full cross validation was used. The PCA was 
carried out for control cells, cells treated with $10 \mathrm{mM} \mathrm{NaB}$ and fast-growing cells. The D-PLS regression was carried out for proteome data from growing cells and apoptotic cells to look for differentially regulated protein spots. Protein spots contributing least to the D-PLS model were removed by Jackknifing through variable selection, and based on the remaining spots significant $(\mathrm{P}<0.05)$ regression coefficients were identified.

\section{RESULTS}

\section{Cell cultures}

The microscopic appearance of Caco-2 cells treated with $\mathrm{NaB}(10 \mathrm{mM}), \mathrm{FCS}$ $(10 \%)$, or in basal medium alone is shown in Figure 1. Cells treated with $\mathrm{NaB}$ $(10 \mathrm{mM})$ appeared apoptotic as indicated by condensed chromatin and fragmented nuclei (Figure 1c). Cells cultured in 10\% FCS showed a very dense cell layer leaving essentially no space in between cells (Figure 1b) compared with control cells (Figure 1a).
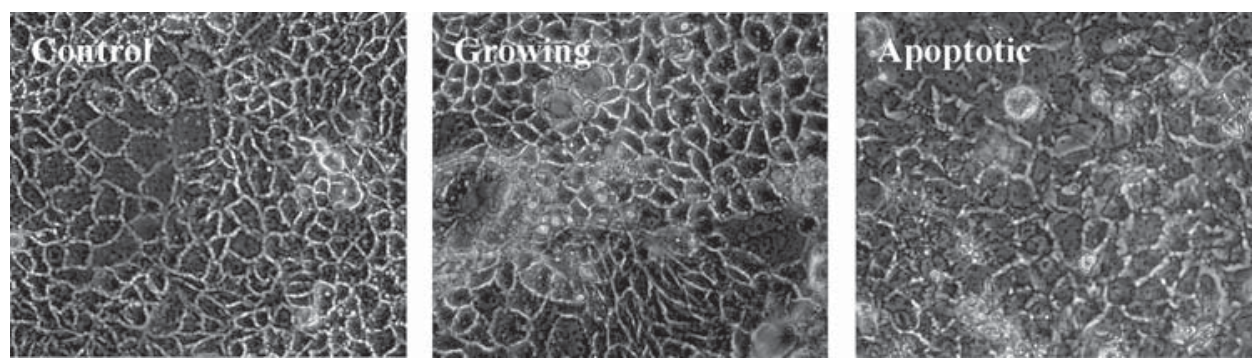

Figure 1. Microscopic appearance of human adenocarcinoma (Caco-2) cells cultured for $72 \mathrm{~h}$ in medium containing $0.625 \%$ FCS (control), $10 \%$ FCS (growing) or $10 \mathrm{mM} \mathrm{Na-butyrate} \mathrm{(apoptotic)}$

Increasing $\mathrm{NaB}$ caused a biphasic response in cell proliferation (Figure 2). Concentrations of 0.1 and $1 \mathrm{mM}$ of $\mathrm{NaB}$ increased cell proliferation by 18 and $34 \%$, respectively, while concentrations of 5,10 and $50 \mathrm{mM}$ decreased cell proliferation to 77,41 and $12 \%$, respectively, compared with proliferation in control cells. Treatment of cells with $10 \%$ FCS increased cell proliferation by $55 \%$ compared with the control cells (data not shown). Cell apoptosis measured as caspase $3 / 7$ activity was lower in cells treated with $10 \%$ FCS compared with control cells $(365 \pm 11$ vs $515 \pm 15 \mathrm{AU})$, while apoptosis in cells treated with $10 \mathrm{mM} \mathrm{NaB}$ was increased by a factor more than 8 relative to apoptosis in control cells (4444 \pm 34 vs $515 \pm 15 \mathrm{AU})$. 


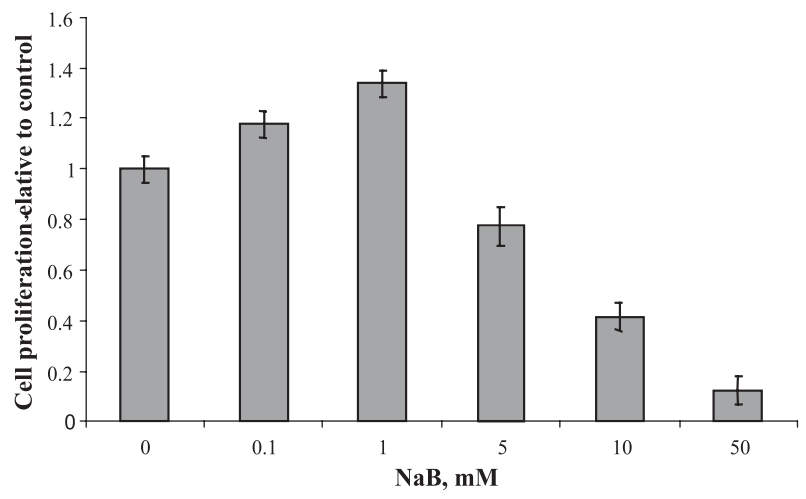

Figure 2. Effect of Na-butyrate $(\mathrm{NaB})$ on proliferation of human adenocarcinoma (Caco-2) cells measured by alamarBlue reduction. Cells were cultured for $72 \mathrm{~h}$ in medium containing different concentrations of $\mathrm{NaB}$. Values are least square means obtained from 2 culture experiments with 4 replicates and presented as relative to proliferation obtained in basal medium

\section{Proteomics}

A typical gel of the separated proteins in the Caco-2 cell lysates is shown in Figure 3. Image analysis of the 2D-gels in the analysis included annotation of 571 individual protein spots.

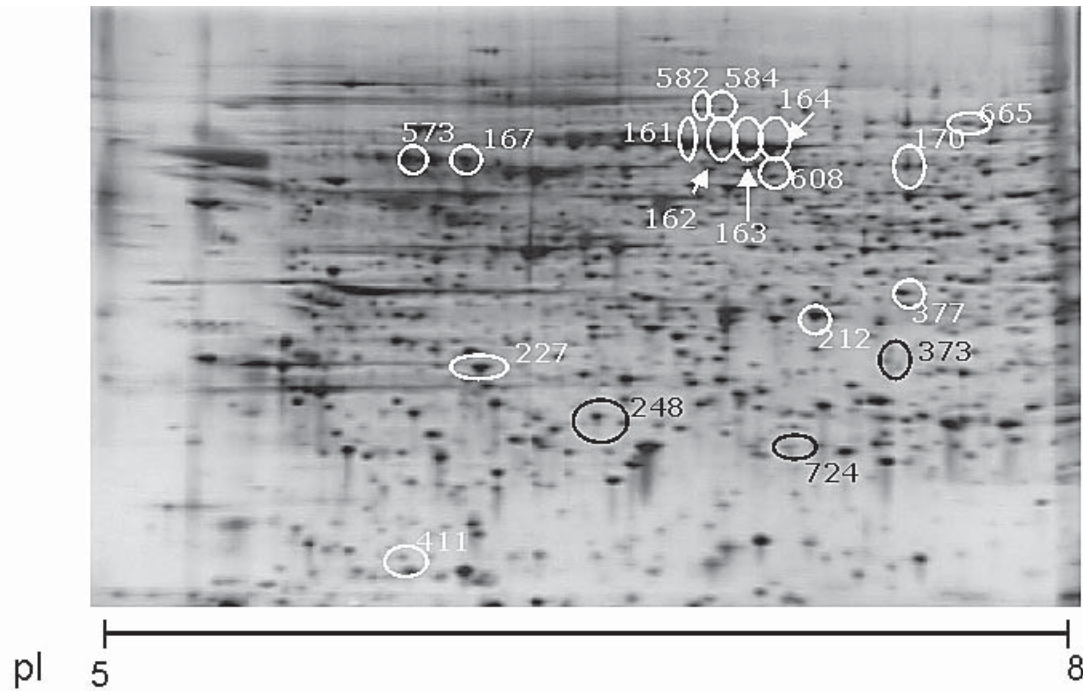

Figure 3. 2-DE of proteins in Caco-2 cell lysates. The $\mathrm{pH}$ gradient of the first dimension is indicated. The ID's of proteins up-regulated in $\mathrm{NaB}$ treated cells (black) and fast-growing cells (white) according to the image analysis are indicated 
Multivariate statistics using PCA of relative spot values showed that the protein expression in NaB-treated cells was different along the PC2 axis from both control cells and fast-growing cells, which in turn were more similar in their protein expressions (Figure 4). The grouping along the PC1 axis was mainly due to variations between two different experiments.

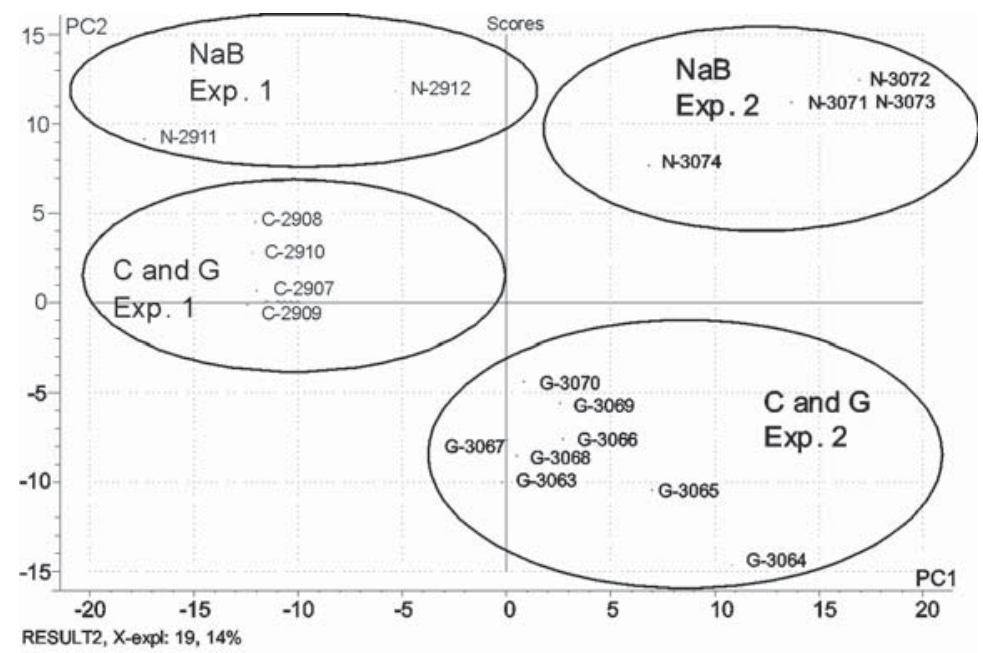

Figure 4. PCA score plot of 2DE-gel samples along the first two PCs. $\mathrm{N}$ indicates $\mathrm{NaB}$ treated samples, while $\mathrm{C}$ and $\mathrm{G}$ indicate control cells and fast growing cells, respectively. The numbers are ID's of 2DE gels

The differences in protein expression between cells treated with $\mathrm{NaB}$ and $10 \%$ FCS (fast-growing cells) were further analysed by D-PLS. The result of this analysis is indicated in Figure 3. A total of 18 protein spots were significantly different between the two treatments. Of these, 15 were down-regulated in the NaB-treated cells, while 3 were up-regulated after $\mathrm{NaB}$ treatment. These significant protein spots were excised from preparative 2D gels, and subjected to MALDI-TOF MS for identification.

By this analysis the following proteins were identified. The accession numbers in SwissProt are indicated. Down-regulated at NaB treatment: spot 167 and 573: $\alpha$-tubulin (P68361); spot 170: T-complex protein $\gamma$-subunit (P49368); spot 212: $60 \mathrm{~S}$ acidic ribosomal protein (Q95140); spot 227: chloride intracellular protein (O00299); spot 377: transaldolase (P37837); spot 584: ATP dependent DNA helicase 2, subunit 2 (P13010); spot 608: Ser/Thr protein kinase (Q8CIN4) and spot 665: stress-induced prosphoprotein 1 (P31948). Up-regulated at NaB treatment: spot 248: ubiquitin C-terminal hydrolase (P09936); spot 373: translin associated protein X (Trax) (Q99598). For two spots, one down-regulated by $\mathrm{NaB}$ 
treatment (spot 411) and one up-regulated by $\mathrm{NaB}$ (spot 724) it was not possible to obtains MS results for identification.

\section{DISCUSSION}

The ability of specific nutrient components to modify the risk of a number of diseases, including cancer, has been the subject of intense studies by nutritional, medical and food researchers. One such nutrient, butyrate, can be derived in large quantities from bacterial fermentation of dietary fibre in the bowel, and can also be found in milk. Since butyrate has been shown to induce apoptosis in cancer cells, it may contribute to the decreased incidence of bowel cancer that has been associated with fibre intake.

The effects of butyrate on the epithelial cells of colon have been examined in a number of in vitro cell-based models. These studies have shown that the cellular effects of butyrate are complex, especially since those observed in normal and transformed (cancerous) colon epithelial cells might be opposite (Archer and Hodin, 1999; Dzierzewicz et al., 2002). Using integrated proteomics and biological assays, we characterized the effect of butyrate on human colonic adenocarcinoma (Caco-2) cells.

The biological assays showed that butyrate decreased cell proliferation in agreement with previous studies (Dzierzewicz et al., 2002; Fu et al., 2004). This decrease was seen at physiological concentrations of $\mathrm{NaB}$ (i.e. 5 and $10 \mathrm{mM}$ ) and at higher concentrations of $\mathrm{NaB}$ (i.e. $50 \mathrm{mM}$ ). However, lower concentrations of $\mathrm{NaB}$ (i.e. 0.1 and $1 \mathrm{mM}$ ), increased cell proliferation compared with control cells. As the concentration of butyrate in the human colon varies from 5-25 mM (Bergman, 1990; Fusunyan et al., 1998), an inhibitory effect of butyrate on colon cells might be suggested in human colonic epithelial tissue. Butyrate has furthermore been shown to be a differentiating and apoptotic agent in Caco-2 cells (Dzierzewicz et al., 2002). In the present study, apoptosis, as measured by caspase $3 / 7$ activity, was increased substantially by $\mathrm{NaB}$ added to cells in a concentration of $10 \mathrm{mM}$. These results suggest that butyrate, as a luminal component, may influence the balance between cell proliferation, differentiation and apoptosis, required for maintaining the health of the large bowel epithelium.

Using proteomics as a tool, we took the characterization of the effects of butyrate on the protein expression patterns one step further. The protein expression pattern of NaB-treated Caco- 2 cells was compared with protein expression in fastgrowing and control Caco-2 cells. The identified proteins down-regulated at $\mathrm{NaB}$ treatment included cytoskeleton proteins, enzymes, chaperones and regulatory proteins. A protein involved in ubiquitination of proteins for the degradation by proteasomes was up-regulated in the apoptotic $\mathrm{NaB}$-treated cells. However, translin 
associated protein $\mathrm{X}$ (Trax), a protein essential for cellular proliferation (Yang and Hecht, 2004), was surprisingly up-regulated by $\mathrm{NaB}$ treatment. The present data are in accordance with the suggested mechanism for effect of butyrate on cells, where the cellular cytoskeleton has been identified as a major target by modifying microfilament and microtubule assembly (Hague and Paraskeva, 1995).

\section{CONCLUSIONS}

The present study shows that butyrate inhibit proliferation and induce apoptosis in human adenocarcinoma (Caco-2) cells. These data are in line with the epidemiological and experimental data suggesting that a diet high in fibres protects against the development of colon cancer. Proteomics showed that the protein expression was significantly affected by $\mathrm{NaB}$ treatment, and that cytoskeletal proteins and chaperones were higher in fast-growing cells, while a protein involved in protein degradation by chaperones was up-regulated by $\mathrm{NaB}$. It is concluded that $\mathrm{NaB}$ has significant effects on proliferation and apoptosis of human intestinal cancer cells and that these effects are reflected in the protein expression patterns of the cells.

\section{ACKNOWLEDGEMENTS}

The authors thank Hanne S. Møller and Annette K. Nielsen for their skilled technical assistance. Jan T. Rasmussen, University of Aarhus, is acknowledged for providing Caco-2 cells.

\section{REFERENCES}

Archer S.Y., Hodin R.A., 1999. Histone acetylation and cancer. Curr. Opin. Genet. Develop. 9, 171-174

Bergman E.N., 1990. Energy contributions of volatile fatty acids from the gastrointestinal tract in various species. Physiol. Rev. 70, 567-590

Dzierzewicz Z., Orchel A., Weglarz L., Latocha M., Wilczok T., 2002. Changes in the cellular behaviour of human colonic cell line Caco-2 in response to butyrate treatment. Acta Biochim. Pol. 49, 211-220

Fu H., Shi Y.Q., Mo S.J., 2004. Effect of short-chain fatty acids on the proliferation and differentiation of the human colonic adenocarcinoma cell line Caco-2. Chin. J. Digest. Dis. 5, 115-117

Fusunyan R.D., Quinn J.J., Ohno Y., MacDermott R.P., Sanderson I.R., 1998. Butyrate enhances interleukin (IL)-8 secretion by intestinal epithelial cells in response to IL-1 $\beta$ and lipopolysaccharide. Pediat. Res. 43, 84-90 
Hague A., Paraskeva C., 1995. The short-chain fatty acid butyrate induces apoptosis in colorectal tumour cell lines. Eur. J. Cancer Prev. 4, 359-364

Jensen O.N., Larsen M.R., Roepstorff P., 1998. Mass spectrometric identification of and microcharacterization of proteins from electrophoretic gels: Strategies and applications. Proteins - Struct. Funct. Genet. 33 (S2), 74-89

Jensen R.G., 2002. The composition of bovine milk lipids: January 1995 to December 2000. J. Dairy Sci. $85,295-350$

Lametsch R., Bendixen E., 2001. Proteome analysis applied to meat science: characterizing post mortem changes in porcine muscle. J. Agr. Food Chem. 49, 4531-4537

Lametsch R., Roepstorff P., Bendixen E., 2002. Identification of protein degradation during postmortem storage of pig meat. Agr. Food Chem. 50, 5508-5512

Shevchenko A., Wilm M., Vorm O., Mann M., 1996. Mass spectrometric sequencing of proteins from silver stained polyacrylamide gels. Anal. Chem. 68, 850-858

Yang S., Hecht N.B., 2004. Translin-associated protein X is essential for cellular proliferation. FEBS Lett. 576, 221-225 